\title{
FUTURE DEVELOPMENT OF SECURITY PRINTING AND RFID MARKS
}

\author{
Iskren Spiridonov (iD, Kosta Shterev (iD, Tatyana Bozhkova \\ University of Chemical Technology and Metallurgy, \\ Department of Printing Arts, Pulp and Paper, Sofia, Bulgaria
}

\begin{abstract}
In the current research are described various ways to protect secure documents, labels and packages, and the different security printing techniques applied today and the way they will evolve in future. Security end products constitute a wide variety of different types of documents, packages, labels and cards. The degree to which these need protection from counterfeiters, forgers and terrorists depends upon how deleterious their illegal procurement and misuse is to human welfare and financial stability. In order to reduce forgery and counterfeiting, the printing industry uses a big range of variable security methods and via different combinations, a high security document is achieved. Security printing devices are often categorized as overt, covert and machine-readable.

Another advantage that the security printer has today is the ability to produce security documents on nontraditional, nonporous substrates such as plastic and polymer - biaxially oriented polypropylene. Due to this complexity in its chemical structure documents and banknotes have a greater abrasion resistance and lower rate of tampering. Our research will aim not only to configure well known methods of security printing arts, but as well get a deeper view of what is coming up next and how for example could the RFID marks become an affordable and reliable security features of our future production.
\end{abstract}

Key words: security printing, substrates, inks, printing technologies, RFID

\section{INTRODUCTION}

Security printing is the field of the printing industry that deals with the printing of items such as banknotes, cheques, passports, tamper-evident I labels, product authentication, stock certificates, postage stamps and identity cards. The main goal of security printing is to prevent forgery, tampering, or counterfeiting.

Different types of printing techniques are suited to different applications. Sometimes a heavier weight of ink is needed on the substrate in order for the security feature to work properly. In other instances is needed to use a technique that uses a more delicate approach, so that the detailing in the artwork adds to the security.

In recent days and in an overgrowing market, the industry of Security printing is struggling to bring up new ideas, with more security features and less coasts. Vital parts in Printing Industry play the latest approaches in the field of chemistry. The ability to produce security documents on nontraditional, nonporous substrate such as plastic and polymer is not anymore a futuristic technology, but an adequate solution in several spheres (Warner et al, 2005).

\section{METHODS IN SECURITY PRINTING}

\subsection{Substrates in Security Printing}

Substrates for printing are mainly paper, polymer and cardboard. Security substrates can be designed and manufactured with special features and considerations to protect documents from counterfeiting or alteration of information (forgery). On Figure 1 there is a classification shown on both types of substrates used in security printing - mainly on banknotes. In some specific cases, substrates can be personalized and produced with certain features and unique securities in order to improve the level of protection and to prevent from counterfeiting and forgery. Most banknotes are made of heavy paper, almost always from cotton fibers for strength and durability, in some cases linen or specially coloured or forensic fibers are added to give the paper added individuality and protect against counterfeiting. Some countries, including Nigeria, Romania, Mexico, New Zealand, Israel, Singapore, Malaysia, United Kingdom and Australia, produce polymer (plastic) banknotes, to improve longevity and allow the inclusion of a small transparent window (a few millimeters in size) as a security feature that is difficult to reproduce using common counterfeiting techniques (Izdebska et al, 2015). Polymer banknotes are banknotes made from a polymer such as bi-axially oriented polypropylene (BOPP). Polymer banknotes last significantly longer than paper 
notes, causing a decrease in environmental impact and a reduced cost of production and replacement. In November 2011 Canada joined the list of countries using polymer currency as it began the introduction of a new banknote series (Polygrafia Magazine).

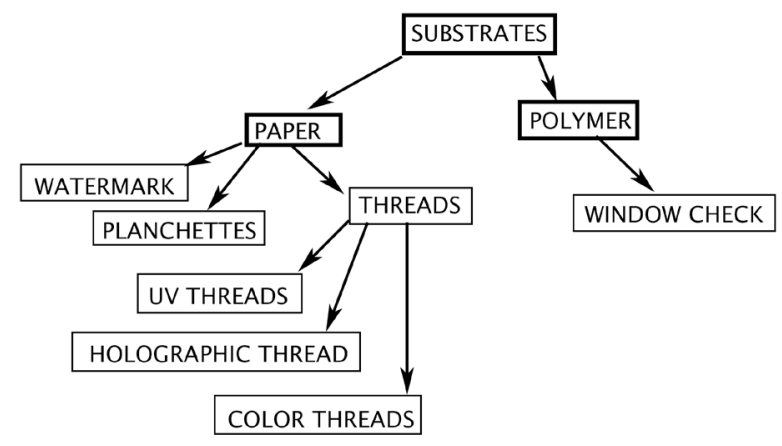

Figure 1: Security features in substrates

\subsection{Security inks}

A wide variety of security inks can be used in documents, packages, labels, and cards. In addition to security printing inks, the printer can also use overprint varnishes and laminates to help deter counterfeiting. It should be noted that many security printing inks depend upon the absorption of UV radiation and its re-emission as visible light. Therefore, to work properly, many security designs and devices must be printed on UV-dead or uncoated paper. On other media they will only work if there are no UV brighteners in the substrates (Vuarnoz et al, 2003):

- $\quad$ Fluorescent dyes;

- Iridescent inks;

- Photochromic ink;

- Phosphorescent inks;

- Thermochromic ink;

- Optical variable ink;

- Infrared ink;

- Machine readable inks;

On Figure 2 can be observed most of the security inks, divided in three branches: overt, covert, semi-covert.

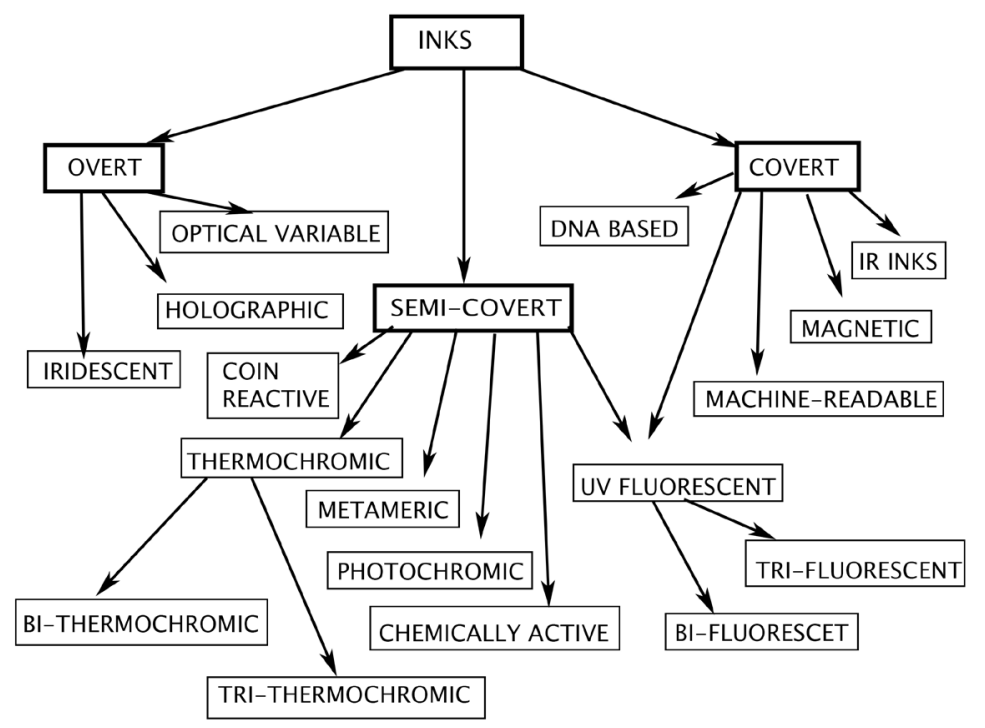

Figure 2: Security features within inks 


\subsection{RFID hidden security features}

RFID, which is an acronym for Radio Frequency Identification, is not a new technology. It was first used in the late 1960's, but it has only become more widespread with advances in technology.

RFID Systems consist of a transponder, also known as a tag, which is basically a microchip connected to an antenna. The tag is mounted to an item, such as a pallet of goods in a warehouse, and a device called a reader communicates with the tag via radio waves. Depending on the type of tag that is used, the reader can receive detailed information or it can receive data as simple as an identification number.

RFID is similar to barcode systems in which data, such as a price, is accessed when the barcode is read. The main difference is that the barcode must come in direct contact to an optical scanner/reader and the RFID tag can transmit to the reader via radio waves and does not have to be in direct contact. An RFID reader can receive data from as many as 1,000 tags per second.

The radio signals can go through many non-metallic substances such as rain, fog, snows, dirt and grime, painted surfaces, etc. This gives RFID tags a distinct advantage over optically read items, such as barcodes, which would be useless under similar conditions (Vig, 2007; Khan, 2014; Printing Knowledge). The many uses for RFID technology include:

- Smart labels and security labels

- $\quad$ Product and inventory management

- RFID chips in car keys for security

- Theft control

- Placement on pharmaceuticals to prevent counterfeited drugs from entering the legal supply chain

- Increased efficiency in admissions into entertainment or sporting events

- Increased efficiency in toll road payments

- Monitoring the whereabouts of luggage, library books, livestock, etc.

It is predicted that RFID use will continue to increase. It is unlikely to ever be as cost-effective as barcoding, but it will become dominant in areas where barcoding and other optically read technologies are not effective.

\section{RFID Tag Categories}

The basic types of RFID tags can be classified as read/write and read only. The data stored on read/write tags can be edited, added to, or completely rewritten, but only if the tag is within the range of the reader. The data stored on a read only tag can be read, but cannot be edited in any way. Read/write tags are much more expensive than read only tags, so they are not used for tracking most commodity items. RFID tags are further categorized as:

- Active tags, which contain a battery that powers the microchip and allows it to transmit a signal to the reader.

- Semi-active (or semi-passive) tags, which contain a battery to run the circuitry of the chip, but must draw power from the magnetic field created by the reader in order to communicate with the reader.

- Passive tags, which rely solely on the magnetic field created by the radio waves sent out by the reader to create a current that can be received by the antenna within the passive tag (Printing Knowledge)

RFID Tag Shapes and Sizes

RFID tags can be manufactured in several different shapes and sizes depending on the type of application in which they will be used.

- $\quad$ Some are the size of a pencil lead or are less than a half-inch in length and can be inserted under the skin of animals and livestock.

- Screw-shaped tags are used to identify specific trees.

- Rectangular RFID tags found in some products are used as an anti-theft device.

- Large, heavy duty tags that are several inches in length, width, and depth are used to track large containers or large vehicles such as trucks or rail cars. 


\section{Printing}

With most types of printed applications, such as labels, the user is unaware of the existence of the chip and antenna because of the different methods of concealing them on the document. Some printers (such as label printers) purchase inlays (containing the RFID) that are already manufactured and then incorporate them into their printed products.

\section{FUTURE TRENDS IN SECURITY PRINTING AND RFID TAGS}

With the overgrowing markets and needs of greater security features, RFID tags can be evolved in taking care for the high security of shares. Since a single share presented on a special piece of paper can be the key to a big amount of money and thus to become an attractive product for counterfeiting. RFID tags in several ways can improve the ranking of security end product and at a descent price for production can save a lot of trouble and inconvenience.

There are several ways to achieve this security feature but at a reasonable price a more adequate roll will play to the top the passive tags - they which rely solely on the magnetic field created by the radio waves sent out by the reader to create a current that can be received by the antenna within the passive tag (Wikipedia, 2018; Printing Knowledge). Components of passive RFID system:

- An antenna is attached to a microchip.

- The antenna allows the chip to transmit information to a reader, which also has an antenna.

- The reader is the device that actually sends out the radio waves to create a magnetic field. A passive RFID tag draws its power from this magnetic field, which powers the circuits in the microchip allowing it to transmit data back to the reader.

- Reader transmits to a computer system.

- The computer passes data onto a network.

- Software determines how the data received should be used (Printing Knowledge)

These passive RFID tags (shown on Figure 3) are much cheaper and can be pre-ordered and attached as a hidden label on the share already printed sheet of paper, or in search for a greater security, they can be ordered built in the paper, ready to print over.

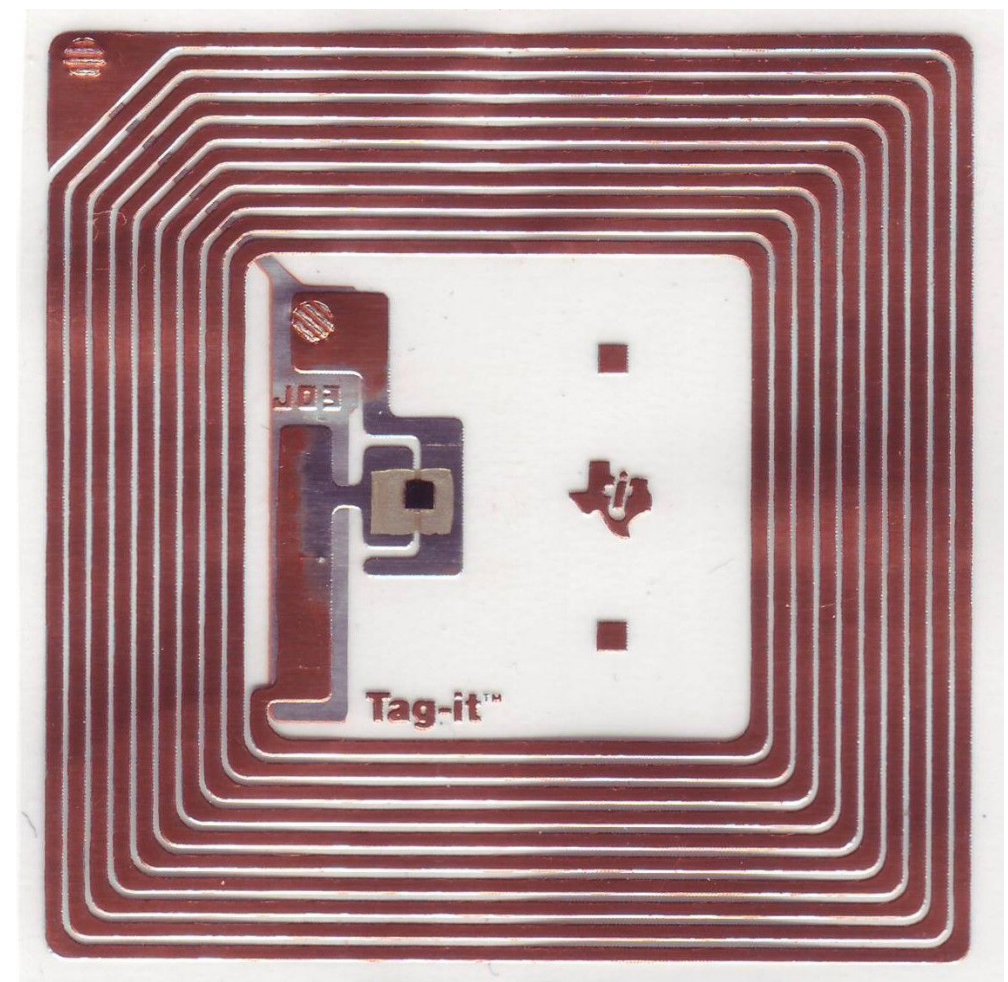

Figure 3: Passive RFID Tag 
After having the shares printed and with an RFID tag already built in (Figure 4), the further need is only to prepare the RFID tag reader. This can automatically prevent the needs of eye checks for watermarks and other common security features for high end security products that can be manipulated easily.

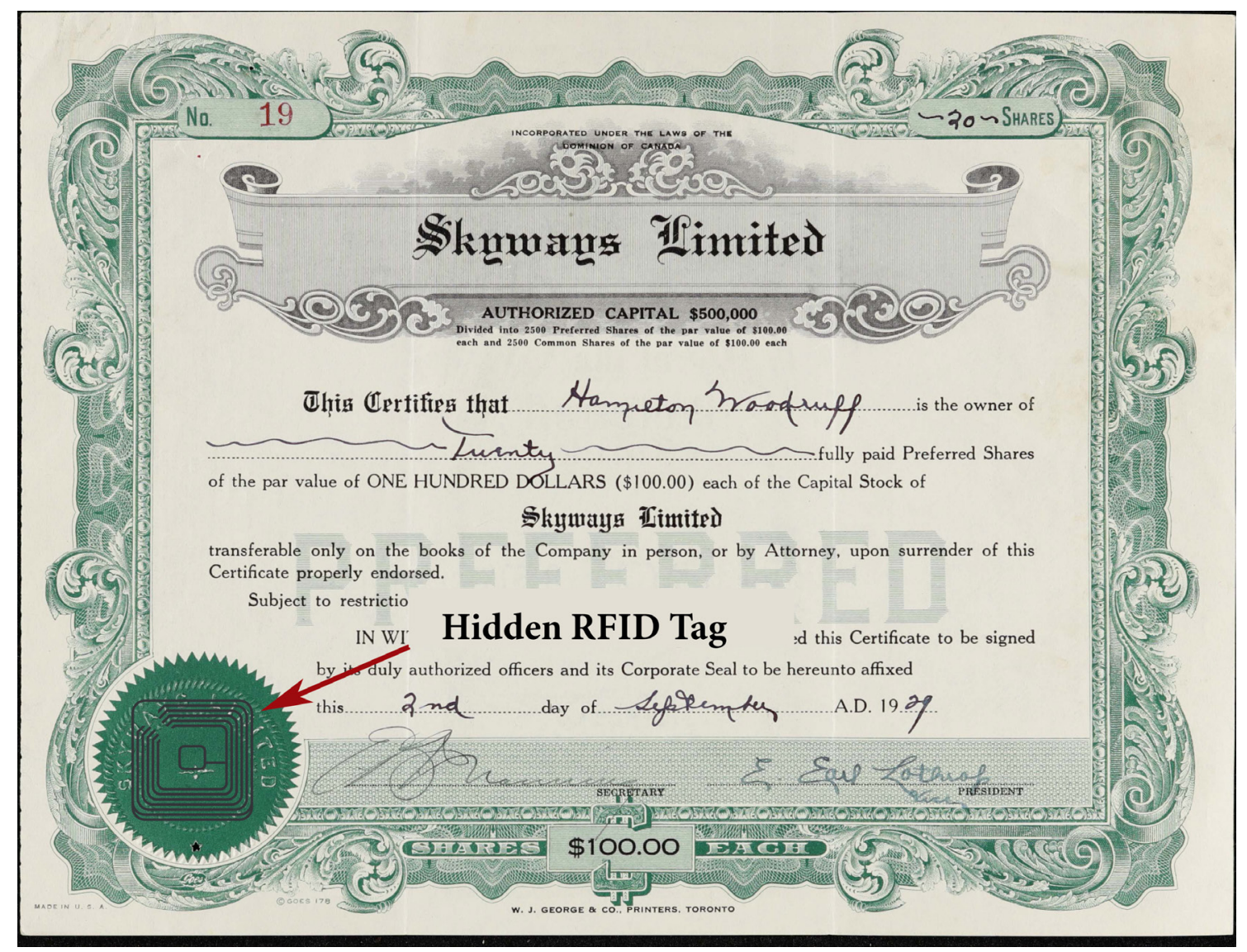

Figure 4: Hidden RFID Tag in Shares

\section{CONCLUSSIONS}

In the high demand of security and an the more creative counterfeiting methods for gaining fortune in an illegal way, our society should move to a higher level of security to any of the common day branches and try its best to protect the welfare of our products in order to preserve stable growth in markets.

In our research we have followed the how process of security features in high end products and the trends to its future development. On a daily basis shares are not an everyday product, that will be used, but their value and the cost of its forgery and counterfeiting can become a great loss for its holder. Using machine-readable methods for shares in order to prevent counterfeiting will be a new method for this sphere in the printing industry. A slight rise of production price but a greater security level can be achieved by implementing a passive RFID tag within each share stock blanc itself thus a reader can at first check tell if the shareholder owns an original share.

Moving on to the next level of security in any kinds of security documents and products is mandatory in order to stay one step ahead of criminals. RFID tags can provide vast possibilities to achieve it.

\section{REFERENCES}

[1] Izdebska, J., Sabu, T.: "Printing on Polymers Fundamentals and Applications", (Elsevier Inc., Amsterdam, 2016).

[2] Khan, S., Lorenzelli, L., Dahiya, R.: "Technologies for printing sensors and electronics over large flexible substrates: a review”, IEEE Sensors Journal, 15 (6), 3164-3185, 2014.

doi: 10.1109/JSEN.2014.2375203 
[3] Polygrafia Magazine, "Security Elements used in the printing of banknotes", URL: http://polygrafiamagazine.bg/ (last request: 2018-10-21)

[4] Printing Knowledge, Security Features | RFID, URL: http://www.printingknowledge.com/securityfeatures-rfid/

[5] Vig, R.: "General Overview of Overt \& Covert Security Features for Brand Protection in Inks and Coatings", URL: http://www.tappi.org/content/events/07place/papers/vig.pdf (last request: 2018-10-21)

[6] Vuarnoz, A., Amrein, O., Veya, P.: Patent - WO2003020834A1, "Ink composition comprising optically variable pigments, use of the composition, optically variable pigment and method of treating said pigment, 2003.

[7] Warner, R. D., Adams, R. M.: "Introduction to Security Printing", (PA: PIA/GATF Press, Pittsburgh), Chapters 1-2;4-9

[8] Wikipedia: "Security Printing", URL: https://en.wikipedia.org/wiki/Security_printing (last request: 2018-10-21).

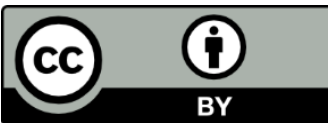

(C) 2018 Authors. Published by the University of Novi Sad, Faculty of Technical Sciences, Department of Graphic Engineering and Design. This article is an open access article distributed under the terms and conditions of the Creative Commons Attribution license 3.0 Serbia (http://creativecommons.org/licenses/by/3.0/rs/). 